\title{
Conformación de la microempresa marginada en la frontera norte de México
}

\author{
José Aguilar Barceló \\ Natanael Ramírez Angulo* \\ Karla Barrón Arreola**
}

Resumen. A través de algunas técnicas de análisis multivariante, particularmente los análisis de conglomerados y discriminante, se estudian 174 microempresas marginadas en el giro de alimentos y bebidas en la zona fronteriza de Baja California, a fin de determinar las características que rigen las decisiones propias de su funcionamiento. Los resultados muestran comportamientos diferenciados de los microempresarios de acuerdo con su edad, condición familiar, educación y zona geográficas de procedencia. Sin embargo, sobresale la relevancia de la educación como determinante de la calidad de la gestión de la empresa y a su vez como una señal en el entorno que atrae la confianza tanto de los proveedores como clientes. Por otro lado se hace patente que el financiamiento, bajo ciertas circunstancias, podría ayudar a mejorar la eficiencia de muchas de estas microempresas. Palabras clave: microempresas, marginación, comportamiento, análisis de conglomerados, análisis discriminante.

Abstract. Using some methods of multivariate analysis, particularly the cluster and discriminate analysis, we study 174 microcompanies and/or microbusiness mainly dedicated to the elaboration of foods and drinks. They belong to the marginalized segment of entrepreneurs in the Mexican border state of Baja California. We wanted to know how they behave and what the variables that define this behavior are. The results show a differentiated behavior among entrepreneurs depending on variables as age, familiar condition, education and geographic zone of origin. Nevertheless, we must emphasize the relevance of education in the quality of the management of the company as well as a signal in the market that bring up confidence to suppliers and clients. On the other hand, we corroborate that microcredits could help to improve the efficiency of many of these microcompanies and/or microbusiness under certain circumstances.

Keywords: microcompanies, microbusiness, marginality, behavior, cluster analysis, discriminate analysis.

* José Aguilar Barceló y Natanael Ramírez Angulo son profesores de tiempo completo de la Facultad de Economía y Relaciones Internacionales de la UABC. Correos electrónicos: gaba@uabc.mx,natanael@uabc.mx

** Técnico académico de tiempo completo de la Facultad de Contabilidad y Administración de la UABC. Correo electrónico: kbarron@uabc.mx 


\section{Introducción}

La economía mexicana sigue presentando serias dificultades para que una parte significativa de su población pueda encontrar en el mercado laboral una fuente de ingreso estable y suficiente para subsistir, y a consecuencia de ello, una de sus constantes es el autoempleo informal a través de micronegocios.

Estos micronegocios tienen características comunes, entre ellas la falta de planeación en su operación, el haber amortizado ex ante las inversiones en capital o costos fijos indispensables para la producción y la ausencia de barreras a la entrada y a la salida, particularmente aquellas derivadas de trámites y declaraciones ante las autoridades debido a la informalidad en la que operan. ${ }^{1}$

Los propietarios de estos micronegocios también presentan rasgos característicos cuando nos enfocamos a los mercados de las zonas fronterizas. Específicamente para el caso de la frontera de Baja California, estos microempresario son muy heterogéneos en origen y cultura, muchos de ellos han optado por esta posibilidad de generación de ingresos después de haber fracasado en el intento de cruzar a los Estados Unidos y tienen en la industria maquiladora una referencia de ingresos que puede afectar la vida del negocio a mediano plazo.

Apoyándose en el instrumental del análisis multivariante, particularmente en el análisis de conglomerados y en el análisis discriminante, el presente trabajo intenta encontrar los principales determinantes del quehacer microempresarial en Baja California. El documento se organiza de la siguiente manera: en el segundo apartado se revisa brevemente el entorno macroeconómico regional como base del entendimiento del comportamiento microempresarial; en el tercero se hace un análisis del entorno empresarial bajacaliforniano; en el cuarto se muestra un sucinto análisis de la microempresa marginada y su desarticulación teórica y práctica, incluyendo la realidad de estas

\footnotetext{
${ }^{1}$ Con informalidad nos referimos específicamente al hecho de que las microempresas no cuentan con un registro de alta ante la Secretaría de Hacienda y Crédito Público. Este término en ningún caso pretende juzgar el nivel de calidad de los productos, servicios o procesos de la empresa.
} 
empresas en el estado, mientras que en el quinto se detalla la metodología utilizada en el análisis. Los principales resultados se discuten en el sexto apartado, en tanto el último se reserva para las conclusiones y consideraciones finales.

\section{El entorno macroeconómico regional}

Al haber priorizado la aplicación de políticas macroeconómicas de estabilización, apertura comercial y liberalización financiera, las políticas de apertura alineadas a los ideales globalizadores han traído beneficios a los sectores modernos y exportadores de las economías en vías de desarrollo, que se han fortalecido y consolidado en términos locales, regional e intersectorial, pero también se han traducido en costos sociales no presagiados para los sectores de su población que se dedicaban al desarrollo de actividades tradicionales a pequeña escala.

Los esfuerzos recientes por formar cadenas productivas de impacto económico en el estado de Baja California no tienen precedentes. Ejemplo de ello es la conformación, entre otros, de los clusters de las industrias electrónica, automotriz, del software, de productos y servicios médicos o del vino, alcanzando algunos de ellos la categoría de "emblemáticos" por los gobiernos locales, lo cual los catapulta como proyectos prioritarios.

Baste decir que a mediados de 2005 la industria electrónica de Baja California registraba 215 plantas, las cuales generaban aproximadamente 92 mil empleos directos. A su vez, la industria automotriz tenía 55 plantas en la entidad generando en su conjunto alrededor de 22 mil empleos directos (Ochoa, 2005).

No obstante, está también presente el fenómeno de la pérdida de competitividad que durante los últimos años y en ciertos sectores productivos ha venido registrando la frontera norte de México. Tal es el caso de la industria maquiladora, que ha venido perdiendo competitividad ante países como China. ${ }^{2}$ Tan sólo de diciembre de

\footnotetext{
${ }^{2}$ Por ejemplo, se calcula que al menos 424 empresas maquiladoras han cerrado en nuestro país desde octubre de 2000 (Pickard, 2003).
} 
2004 a abril de 2005, Baja California había perdido 2157 puestos de trabajo relacionados con la industria maquiladora, ocupando el primer lugar en decrecimiento del empleo en este sector durante 2005 (Sedeco, 2005). En particular ciudades como Tijuana se han visto sensiblemente afectadas por esta situación, perdiendo $12 \%$ de los empleos de esta clase entre 2001 y $2005 .^{3}$

El cierre reciente de estas plantas maquiladoras ha tenido una repercusión directa en los ingresos de las familias bajacalifornianas a pesar del bajo valor añadido que producen para sus empleados de último nivel (Ruiz y Aceves, 2002).

De acuerdo con Sierra y Serrano (2002), una familia representativa de la frontera de Baja California está compuesta por 4.1 miembros, cuenta con un jefe de familia de aproximadamente 40 años, de sexo masculino $82.4 \%$ de las veces y con estudios de secundaria como mucho. Este jefe de familia trabaja en promedio más de 40 horas a la semana, $68 \%$ de las veces como empleado, y aporta ingresos mensuales de 6500 pesos, los cuales representan $80 \%$ del ingreso familiar.

Debido a la restricción al consumo que ha impuesto esta situación, entre otras razones, han surgido un gran número de microempresas familiares, en muchos casos marginadas, ${ }^{4}$ que nacen como una opción para conseguir y mantener una independencia laboral que asegure los ingresos para cubrir las necesidades básicas (Mungaray, Ramírez y Texis, 2005).

\footnotetext{
${ }^{3}$ En términos generales, los motivos de estos cierres han tenido que ver con las regulaciones fiscales y arancelarias derivadas del Tratado de Libre Comercio, la caída de la demanda en el mercado de Estados Unidos, las ventajas comparativas que ofrecen otros países, y los riesgos propios de las ciudades fronterizas como el alto grado de inseguridad pública (Hualde, 2003).

${ }^{4}$ Las empresas marginadas son aquellas que por lo general han sido relegadas o carecen de apoyo económico, se ubican en zonas marginadas, no llevan control de sus operaciones, sus dueños y trabajadores cuentan con un grado de formación mínimo y sus ingresos son escasos, y en ese sentido son pobres (Mungaray et al., 2005). La gran mayoría de las empresas marginadas son informales, aunque esto no necesariamente aplique en sentido inverso.
} 


\section{Estructura empresarial en Baja California}

Dejando de lado la situación específica de la industria maquiladora descrita en el apartado anterior, durante las últimas décadas Baja California ha sido reconocida como un estado con una actividad económica pujante que en parte se asocia a su cercanía con California, una de las economías más dinámicas de Estados Unidos y del mundo. En los años más recientes, la industria manufacturera ha logrado una contribución muy importante al PIB del estado, la cual para 1999 fue del $22.3 \%$, desplazando incluso las actividades de comercio, restaurantes y hoteles que tradicionalmente habían sido las que mayor participación alcanzaban (Ocegueda, 2003).

Sin embargo, no es el sector industrial el que mayor número de empresas reporta en el estado, sino el de comercio. De acuerdo con el Sistema de Información Empresarial Mexicano (SIEM), en el año 2002 Baja California contaba con 12905 establecimientos en las diferentes ramas de actividad económica, $53.4 \%$ de los cuales eran comercios, seguidos por $24.3 \%$ en el rubro de servicios, en tanto el resto $(22.3 \%)$ se concentraba en el sector industrial.

Para el año 2006, el SIEM registraba 9580 establecimientos en todos los sectores de actividad, donde los establecimientos comerciales tenían una participación de $58.16 \%$, mientras que los servicios lo hacían con $27.15 \%$ y el sector industrial con 14.7 por ciento.

Las cifras revelan, pues, que ha habido un decrecimiento en el número de establecimientos en la entidad, lo cual se observa en el cuadro 1. Es de destacar la tasa de disminución anual de $27.28 \%$ de las microempresas industriales.

El impacto para las empresas ha sido inversamente proporcional a su tamaño, siendo de esta manera la microempresa la que ha absorbido, en su mayoría, esta disminución. ${ }^{5}$

La estructura empresarial del estado se reproduce de manera muy similar en los diferentes municipios que lo componen, aunque sin duda Tijuana es el que concentra la mayor parte de las grandes empresas

\footnotetext{
${ }^{5}$ Probablemente debido a la ausencia de barreras a la salida y al precario estado de este tipo de empresas.
} 
Cuadro 1.

Crecimiento medio anual del número de establecimientos por sector económico en Baja California, 2002-2006

\begin{tabular}{lrccc}
\hline & Industria & Comercio & Servicios & Total \\
\hline Microempresa & -27.28 & -5.81 & -4.46 & -8.07 \\
Pequeña & -6.17 & -1.11 & -6.03 & -4.19 \\
Mediana & -3.27 & 2.39 & -3.55 & -1.53 \\
Grande & -1.84 & -0.34 & -1.93 & -1.53 \\
Total & -16.35 & -5.20 & -4.55 & -7.18 \\
\hline
\end{tabular}

Fuente: Elaboración propia con base en información del sIEM de los años 2002 y 2006.

industriales y en este sentido es el más dinámico. ${ }^{6}$ La estructura empresarial de los diversos municipios del estado revela la importancia de las microempresas y pone de manifiesto la necesidad de impulsar una política pertinente que favorezca su desarrollo para hacerlas más eficientes y consolidarlas como opción de vida de quienes dependen de ellas.

El cuadro 2 muestra que en el ámbito municipal la reducción del número de establecimientos ha tenido el impacto más fuerte en los municipios de Rosarito y Tijuana, registrando tasas de crecimiento promedio anual de $-20.34 \%$ y $-11.54 \%$, respectivamente.

En el caso de Tecate, aunque su número de establecimientos ha disminuido por año en $6.5 \%$ en promedio, todas las empresas, excepto las micro, se han incrementado en número, lo que convierte a esta zona en un municipio que proporciona ventajas estratégicas a los empresarios e inversionistas, entre las que podría estar un menor costo de suelo en comparación a Tijuana, sin estar muy alejado de éste. Finalmente, Mexicali es el municipio que de manera global

\footnotetext{
${ }^{6}$ En la actualidad, $38.8 \%$ de las empresas del estado se concentran en Tijuana y $37.96 \%$ en Mexicali; Ensenada participa con 19.96\%. El 83.8\% de estos negocios son microempresas. El resto de los municipios, Tecate y Rosarito, tienen una representación escasa en la estructura empresarial del estado, pues apenas logran una participación de $2.6 \%$ y $0.63 \%$, respectivamente.
} 
Cuadro 2.

Crecimiento medio anual del número de establecimientos por municipio en Baja California, 2002-2006

\begin{tabular}{lrrrrrr}
\hline & Ensenada & Mexicali & Tecate & Tijuana & Rosarito & Total \\
\hline Microempresa & -4.58 & -2.99 & -11.12 & -13.49 & -20.97 & -8.07 \\
Pequeña & -4.68 & -1.97 & 7.67 & -6.10 & -15.91 & -4.19 \\
Mediana & 0.49 & 0.38 & 10.67 & -3.66 & -6.94 & -1.53 \\
Grande & 0.88 & -3.78 & 15.83 & -1.46 & - & -1.53 \\
Total & -4.27 & -2.82 & -6.50 & -11.54 & -20.34 & -7.18 \\
\hline
\end{tabular}

Fuente: Elaboración propia en base a información del SIEM de los años 2002 y 2006.

ha mostrado una menor pérdida de establecimientos por año con 2.8 por ciento.

El cuadro 2 también permite observar el comportamiento que ha tenido el número de establecimientos por sector en el estado, lo que arroja que entre 2002 y 2006 se ha registrado una caída del 7.2\% promedio anual en el número total de establecimientos; el estrato más afectado ha sido en de las microempresas, que muestran una disminución de más del $8 \%$ promedio anual.

Los registros del SIEM señalan que el número de establecimientos micro ha venido disminuyendo en los últimos años; sin embargo, un importante número de familias bajacalifornianas depende de los ingresos generados por las microempresas aun cuando éstas no estén establecidas formalmente, y por tal situación no aparecen en las estadísticas que generan las diferentes instituciones gubernamentales.

\section{La empresa marginada: desarticulación teórica y práctica}

Lo dicho en secciones anteriores nos da argumentos para apoyar la idea de que las microempresas marginadas surgen como mecanismo para atender las necesidades más básicas de sus propietarios antes que como manifestación de un puro espíritu empresarial, es decir, la razón de ser de muchas de estas empresas es la subsistencia. 
De acuerdo con Alcalá, De la O y Hernández (2002), no se cuenta con resultados robustos sobre el comportamiento y evolución de las microempresas, mucho menos de las del tipo "marginado", debido a que éste no se ajusta al enfoque tecnológico, bajo el cual la empresa es simplemente una función de producción que ignora cuestiones organizativas internas o el grado de integración de la actividad productiva (Segura, 1993). La empresa marginada parecería más bien ajustarse al comportamiento resultante de la maximización de algún tipo de "nivel de subsistencia" vinculado al entorno personal-familiar de su promotor, término definido por Cariola y colaboradores (1989) como aquel nivel en el cual se cubren apenas ciertas necesidades básicas de manutención como la alimentación, los gastos de vivienda y sus servicios, transporte, educación y salud.

En definitiva, la correcta definición del problema de estas empresas tendría que ser más cercana a su razón de ser, la cual se relaciona con la necesidad de subsistencia en un mercado interno deprimido y con la ausencia en muchos casos de cualquier tipo de espíritu emprendedor; de hecho, estas empresas suelen estar inmersas en un deterioro sistemático que se manifiesta en la progresiva desacumulación de capital y en una nula capacidad crediticia que genera un escenario de falta de liquidez y solvencia para hacer frente a sus compromisos (Mungaray y Ramírez, 2004).

\section{Metodología}

Como respuesta a la falta de consenso acerca de una teoría que explique el comportamiento de la microempresa familiar y marginada, el presente documento busca, por medio de algunas herramientas del análisis multivariante, encontrar los determinantes del quehacer microempresarial en la zona fronteriza de Baja California.

Lo anterior se consigue por medio de la identificación de patrones de comportamiento de los microempresarios acerca de variables tales como la elección del giro, el grado de adaptación de la maquinaria para ajustarse a la demanda, el tipo de contabilidad llevada a cabo, las facilidades de pago ofrecidas a los clientes y el volumen de com- 
pra de insumos, entre otras decisiones esenciales para el funcionamiento de sus empresas.

En este trabajo, el tamaño de la muestra se determinó con base en un método no probabilístico en virtud de la informalidad, marginación e inestabilidad de estos comercios y sus actividades productivas que hace imposible conocer con precisión su número exacto y ubicación geográfica (Alcalá et al., 2002). En cualquier caso, la muestra ignora a las empresas formales de cualquier tamaño y la interacción de éstas con las informales.

Utilizando la información generada por el Programa de Investigación, Asistencia y Docencia a la Micro y Pequeña Empresa (PIADMyPE), programa institucional de servicio social profesional de la Universidad Autónoma de Baja California, se estudió el caso de 174 microempresas atendidas por el programa entre 2003 y 2005, en distintas zonas marginadas de los municipios de Tijuana, Ensenada, Mexicali y Tecate, estando $66 \%$ de estas empresas dedicadas a actividades de elaboración y venta de alimentos y bebidas, aunque también están representadas otras actividades como la compra-venta de artículos diversos (18\%), la metalmecánica $(9 \%)$ y servicios varios $(7 \%)$.

Las herramientas del análisis multivariante utilizadas son el análisis de conglomerados y el análisis discriminante. El primero pretende encontrar interdependencias relevantes preponderantemente en datos métricos, mientras que el segundo intenta localizar relaciones de dependencias en datos no métricos.

De manera sucinta, el análisis de conglomerados busca dividir un conjunto de elementos en grupos o clusters, bajo el criterio de que los elementos del mismo grupo sean lo más similares posible y los de grupos diferentes, lo más disímiles posible. Particularmente, el método de conglomerados jerárquico, que es el utilizado en este trabajo, ${ }^{7}$ permite que se puedan agrupar las observaciones de manera gradual hasta llegar a un solo cluster en un caso extremo. Este análisis parte de definir una medida de distancia para crear una matriz de distancia entre la muestra, es decir, la distancia entre un elemento y el resto de la

${ }^{7}$ Esto ha sido así debido a que el tamaño de la muestra es lo suficientemente pequeño para ello. 
muestra, lo que permitiría, en una segunda etapa, agrupar los elementos más próximos en un conglomerado indisoluble a partir de ese momento. Para esta segunda parte es necesario determinar un mecanismo de aglomeración.

De acuerdo con la experiencia previa en el manejo de este tipo de datos, se ha elegido la distancia euclídea al cuadrado (dec) para llevar a cabo el análisis. La dec está dada por

$$
\operatorname{dec}(X, Y)=\sum_{i}\left(X_{i}-Y_{i}\right)^{2}
$$

donde $X$ y $Y$ son las observaciones entre las que se está calculando la distancia y el subíndice $i$ se refiere a la variable bajo análisis.

El método utilizado para conglomerar es el método de vinculación inter-grupos que se denota como $d_{A B}$, el cual permite hacer uso de la información de todos los elementos que componen los dos clusters candidatos a ser fusionados. Lo anterior se hace a partir de la siguiente expresión

$$
d_{A B}=\frac{1}{n_{A} n_{B}} \sum_{i \in A} \sum_{j \in B} d_{i j}
$$

que calcula la distancia promedio existente entre todos los pares de elementos -individuales o conglomerados formados previamente- $A$ y $B$, donde $n_{i}$ se refiere al número de observaciones en el cluster, en tanto $i$ y $d_{i j}$ apuntan a la distancia entre los elementos de los clusters (Uriel, 1995).

En el caso del análisis discriminante, los objetivos principales son la discriminación de grupos y la predicción de elementos en uno de varios grupos previamente definidos. La metodología utilizada fue la técnica de análisis discriminante en su forma explicativa, para tratar de determinar la contribución de cada variable discriminante a la clasificación correcta de cada uno de los individuos. Siguiendo a Uriel (1995), el análisis discriminante consiste en la obtención de combinaciones lineales de la forma

$$
D=\beta_{0}+\beta_{1} X_{1}+\beta_{2} X_{2}+\beta_{3} X_{3}+\ldots+\beta_{k} X_{k}
$$


donde $D$ es la clasificación discriminante, $X_{i}$ es la variable independiente o discriminante $i$, y $\beta_{i}$ es su coeficiente asociado. La estimación de los coeficientes discriminantes $\beta$ supone que hay $G$ grupos, $i=1,2$, $3, \ldots, G$, y que cada uno contiene $n_{i}$ observaciones en $k$ variables independientes, $X_{1}, X_{2}, \ldots, X_{k}$ por lo que $N=\sum_{i=1}^{G} n_{i}$ es el tamaño muestral.

Aunque la naturaleza de la relación entre las variables es distinta, el mecanismo de optimización guarda similitud con el del caso del análisis de conglomerados: se trata de que la variabilidad entre grupos sea lo más grande posible en detrimento de la variabilidad intragrupos.

Finalmente, tomando la derivada parcial de $\lambda$ y estableciéndola igual a cero, obtenemos los coeficientes discriminantes $\beta$ especificados en (3). Los principales estadísticos tomados en cuenta para valorar los modelos propuestos han sido el nivel de significancia, la correlación canónica, la lambda de Wilks y el porcentaje de aciertos, elementos de decisión que se definen en el anexo.

\section{Discusión de resultados}

Con la información proporcionada por el "diagnóstico de la empresa" del PIADMYPE, ${ }^{8}$ se evaluó un amplio conjunto de relaciones relevantes a priori con base en los conocimientos teóricos y empíricos proporcionados por investigaciones anteriores.

La literatura previa se ha encargado de identificar ya algunos esquemas suficientemente sólidos, tales como los de que estas empresas, por su estructura, se suelen enfocar a la elaboración y comercialización de productos artesanales dentro de los cuales el microempresario tiene inclinación por el establecimiento de comercios de los giros de alimentación y metalmecánica (Barrón y Aguilar, 2005), o como el hecho de que la dimensión del mercado de la microempresa la circunscribe a un espacio geográfico más bien fijo en el sentido de que muy poca

${ }^{8} \mathrm{El}$ "diagnóstico de la empresa" es el documento que captura la información general de la empresa, aspectos socioeconómicos y aquellos asociados con la operación de la misma, desde costos y producción, hasta recursos humanos e institucionales. 
producción se vende fuera de la zona de influencia o nicho de barrio donde está establecida (Alcalá et al., 2002, y Ramírez, 2002).

En este trabajo, el análisis de conglomerados permite localizar interdependencias entre las observaciones desde distintas perspectivas. Por ejemplo, desde el punto de vista de las características de las microempresas se identifica un primer grupo de las mismas que son importantes en la proveeduría del gasto familiar (representan alrededor del 50\% del mismo), dedicadas principalmente a la elaboración y distribución de alimentos y bebidas, donde su dueño no tiene educación técnica, pero le resulta relativamente sencillo aprender el oficio mediante la experiencia ganada en trabajos anteriores. Este grupo tiene un peso del $34 \%$ de las observaciones analizadas. Existe un segundo grupo en el que la microempresa es indispensable para cubrir los gastos familiares (representa 95\% del mismo), dedicado principalmente a la compra-venta de productos varios y donde se requieren más habilidades técnicas para un buen desempeño en el mismo; este grupo está representado por $66 \%$ de las observaciones analizadas. ${ }^{9}$

Una clasificación alternativa que considera al microempresario como punto de referencia divide a éste en tres grupos. El primero estaría compuesto por microempresarios jóvenes (de alrededor de 30 años) con estudios técnicos, aunque sin mucha experiencia previa y con una tendencia hacia el giro de elaboración y venta de productos alimenticios. Habría un segundo grupo de empresarios maduros (con una media de 44 años) enfocados a la metalmecánica, también con estudios técnicos, cuya microempresa se ha convertido con mucha probabilidad en la única fuente de ingreso familiar. Existe un tercer grupo de empresarios mayores (con una media de 60 años), sin estudios técnicos, que no llevan ningún tipo de contabilidad ni control de inventarios, dedicados principalmente a la compra-venta de artículos varios y donde el ingreso resulta fundamental para la supervivencia del microempresario que en la mayoría de los casos no tiene dependientes económicos. ${ }^{10}$

\footnotetext{
${ }^{9} \mathrm{El} 28.57 \%$ del total.

${ }^{10}$ El 54.29\% del total.
} 
A través del análisis discriminante se han podido delimitar con mayor precisión algunos de los resultados anteriores y se han encontrado otros nuevos. El soporte estadístico de estos resultados se resume en el cuadro 3 al final de este apartado.

Prueba 1. Se encontró relación entre el hecho de que las empresas realizaran adaptaciones a su maquinaria para facilitar su uso y el volumen de compras que éstas llevan a cabo. ${ }^{11}$ Se encuentra que aquellos microempresarios cuya demanda les permite hacer compras al mayoreo, con mayor frecuencia fuerzan su maquinaria intentando obtener mayores niveles de producción ante la imposibilidad de adquirir maquinaria de mayor capacidad, probablemente por no contar con financiamiento oportuno. ${ }^{12}$ Curiosamente, fue más sólida esta relación que la encontrada entre el adaptar o no su maquinaria y el número de empleados de la empresa o el nivel de estudios del microempresario.

Prueba 2. Los resultados arrojan una relación del tipo de contabilidad utilizado en la empresa con el nivel de estudios del microempresario y el tiempo que tarda en vender el producto. Se encuentra que existe una correlación positiva entre el nivel de estudios del microempresario y la posibilidad de llevar a cabo algún tipo de contabilidad. En particular, aquellos que no terminan la primaria con una mayor probabilidad no llevarán ningún tipo de registro contable. Asimismo, aquellos microempresarios que comparativamente suelen tardar poco en vender su producto, con mayor probabilidad no llevarán registros contables de sus operaciones, probablemente debido a, uno, que el producto genera ingresos más allá de la subsistencia, por lo que el control, aparentemente no es prioritario, o dos, que el poco tiempo dedicado al negocio no invita a invertir una parte significativa del mismo en llevar registros contables.

Prueba 3. Las estimaciones muestran relación del nivel de estudios del microempresario con su edad y el número de dependientes eco-

\footnotetext{
${ }^{11}$ En todas las pruebas se ha considerado como dependiente la primera de las variables mencionadas.

${ }^{12}$ Esto no quiere decir del todo que no existan programas de apoyo a microempresas marginadas, sino que puede incluir la posibilidad de que el mismo empresario no los conozca.
} 
nómicos. Se encuentra que aquellos microempresarios con nivel de carrera técnica, o al menos de bachillerato terminado, suelen ser en promedio 6 años más jóvenes y tener un dependiente económico menos que los que sólo alcanzan secundaria. Este hallazgo es interesante pues podría indicar una tendencia hacia el autoempleo en personas cada vez más jóvenes, con más estudios y sin tantos compromisos familiares.

Pruebas 4 y 5. Se encontró relación entre el tipo de industria a la que un microempresario se dedica y el número de sus dependientes económicos. Se encuentra que aquellos que se dedican a la metalmecánica tienen en promedio dos dependientes económicos más que aquellos que se dedican a montar negocios de alimentos y bebidas. La relación tiene al menos una explicación clara y es el hecho de que aquellos que se dedican a la metalmecánica son en su mayoría hombres y casados, lo que eleva el número promedio de dependientes económicos. Los microempresarios dedicados a bebidas y alimentos son más heterogéneos en su composición. También se encuentra que aquellos que se dedican a la metalmecánica suelen tener una habilidad que se ha venido forjando a través de la escolarización (técnica) y trabajos anteriores, mientras que los dedicados a bebidas y alimentos suelen aprender sobre la marcha o mediante los consejos de familiares y amigos.

Prueba 6. Las estimaciones sugieren relación entre la necesidad o no de financiamiento y el tipo de compras que realiza la empresa. Se encuentra que aquellos microempresarios que compran al mayoreo, con mayor probabilidad manifiestan requerir de financiamiento. La necesidad o no de financiamiento no parece estar relacionada con mantener o no inventarios ni con la antigüedad del equipo de producción.

Prueba 7. Se encontró relación entre la percepción del microempresario sobre la evolución de su negocio y el lugar donde se vende el producto. Se encuentra que aquellos microempresarios que salen a las calles en busca de los clientes perciben con mayor frecuencia que su negocio ha mejorado, mientras que aquellos que esperan a que el cliente llegue a su local o domicilio, con mayor probabilidad sienten que su negocio no ha prosperado desde que lo iniciaron.

Pruebas 8 y 9. Los resultados exhiben relación de la entidad federativa de procedencia del microempresario con su nivel de estudios y la in- 
dustria a la que se dedica. Asimismo, se encontró relación entre el estado de procedencia y el número de hijos del microempresario. En particular, aquellos microempresarios originarios de los estados del norte del país ${ }^{13}$ cuentan en promedio con estudios de preparatoria, mientras que los del centro y sur tienen una educación media de estudios de secundaria. Además, aquellos procedentes del centro y sur muestran mayor interés que los del norte por el establecimiento de negocios de metalmecánica. La segunda relación muestra que los microempresarios provenientes del centro y sur de la república tienen en promedio 1.28 dependientes económicos más que los que proceden del norte.

Prueba 10. Las estimaciones sugieren relación entre el hecho de que los proveedores otorguen o no crédito a la microempresa, con la edad del microempresario y el tipo de contabilidad utilizada en el negocio. Esto nos lleva a comprobar que aquellos microempresarios que llevan algún tipo de contabilidad con mayor probabilidad contarán con el apoyo crediticio de sus proveedores, de ahí la importancia de la asistencia microempresarial en la mejora de la operación y la rentabilidad de estos micronegocios. Además, aquellos microempresarios que cuentan con crédito comercial suelen tener en promedio 7 años más que aquellos que no lo reciben. Dado que existe una correlación positiva entre la edad del microempresario y el tiempo de vida de su negocio, se entiende que este resultado tiene que ver con la estabilidad económica de la microempresa.

Prueba 11. Se encontró relación de la planeación o no de las compras de insumos con el hecho de mantener o no inventarios y el nivel diario de ventas. Aquellos que mantienen inventarios muy probablemente afirmarán que planean sus compras de insumos. Aunque éste puede ser un resultado contraintuitivo para el caso de una empresa de tamaño mediano y grande, preocupada por minimizar el costo de inventario la microempresa se ve empujada a mantener inventarios si quiere aprovechar mejores tarifas y evitar significativos costos de transacción ante la ausencia de relaciones de largo plazo con su entorno comercial.

${ }^{13}$ Entiéndase por estados del norte del país Baja California, Baja California Sur, Sonora, Chihuahua, Nuevo León, Tamaulipas, Sinaloa, Durango y Zacatecas. 


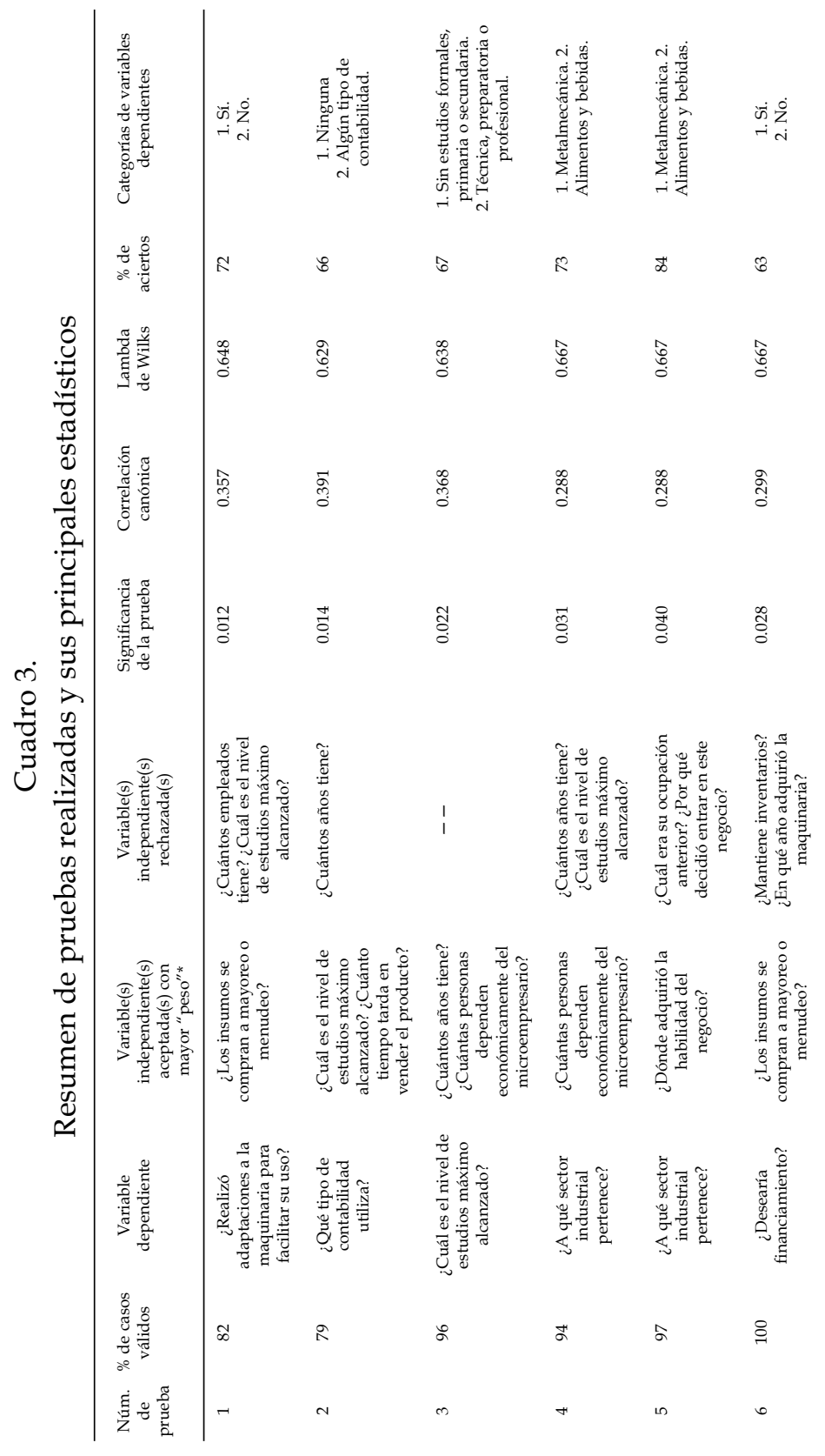




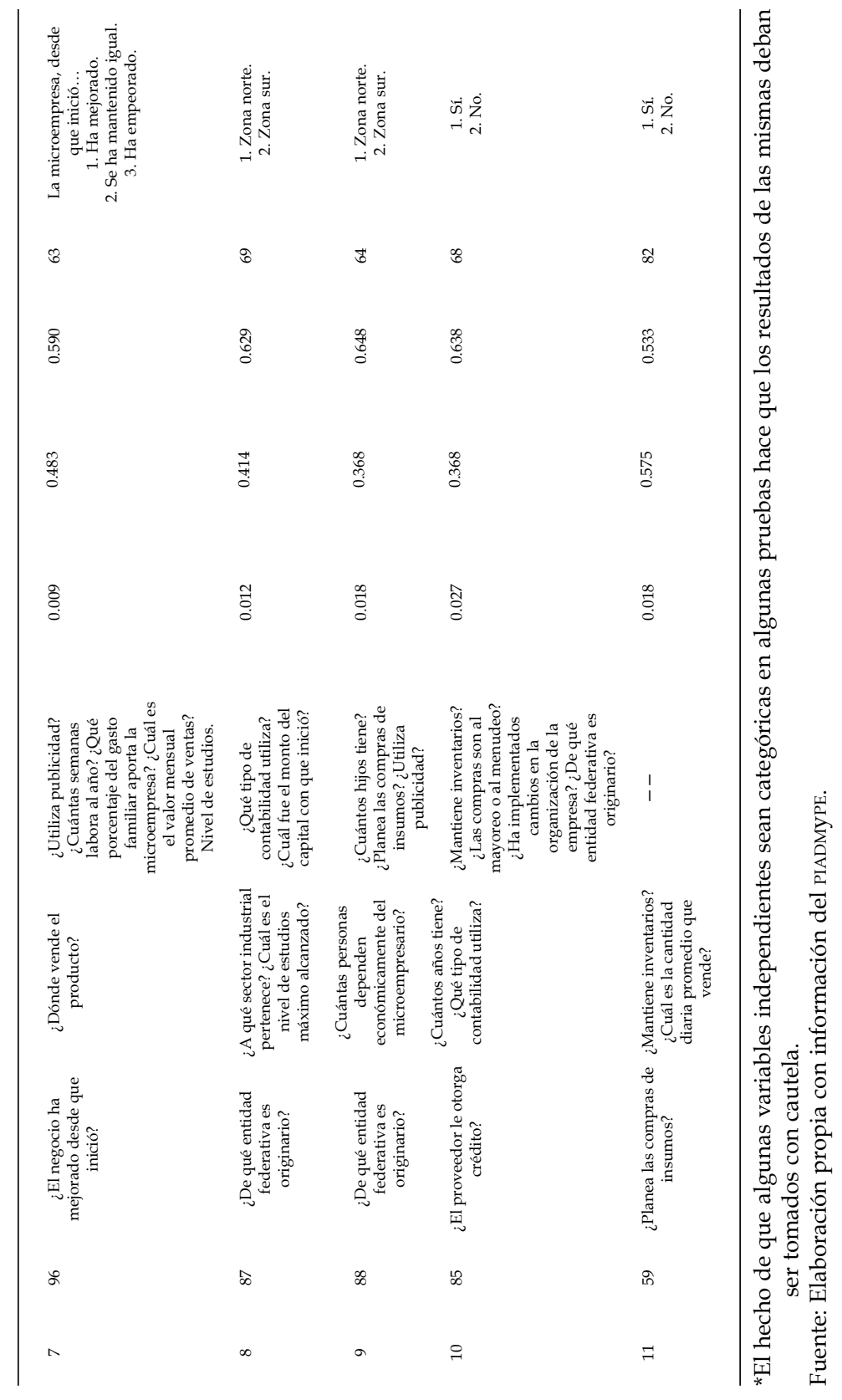




\section{Conclusiones}

A través de las técnicas estadísticas del análisis de conglomerados y del análisis discriminante se analiza una muestra de microempresas marginadas en la zona fronteriza de Baja California con la finalidad de identificar su conformación y patrones de comportamiento en sus responsables.

Los resultados indican que, por ejemplo, es significativa la influencia que ejerce el nivel de estudios, la edad, el número de dependientes económicos y el origen de los microempresarios en las decisiones propias de la empresa, como son el llevar o no registros contables, la elección del giro y la posibilidad de ofrecer opciones de crédito a sus clientes frecuentes. Lo anterior evidencia la necesidad de la asistencia técnica a este tipo de empresas para eficientizar su operación.

Por otra parte, los resultados establecen que las empresas que tienen un mayor nivel de demanda por lo general hacen adaptaciones a sus equipos y llevan a cabo las compras de insumos al mayoreo en un intento de disminuir sus costos medios. No obstante, también se observa que dicho objetivo suele verse frustrado por las necesidades no cubiertas de financiamiento, que en muchos casos se da debido a que la microempresa no califica para el mismo, pero en otros ocurre porque ésta ni siquiera tiene conocimiento de esta posibilidad. En cualquier caso, el apoyo a estas empresas por medio de microcréditos con el objetivo de elevar su producción al nivel del tamaño mínimo eficiente tendría que reforzarse con un conocimiento más profundo de su naturaleza y entorno, pero también con mucha más información del lado de la demanda.

Además, y al margen de las mejoras en eficiencia social y asignativa, los resultados esperados al otorgar apoyos económicos a una empresa marginada generalmente son considerados desde la óptica de que la empresa es equivalente a su función de producción. No obstante, es precisamente en el caso de la microempresa marginada cuando estos términos alcanzan una diferenciación significativa.

Por lo general, las ganancias de las microempresas marginadas son destinadas a cubrir los gastos personales y familiares del promotor, debido a lo cual los factores productivos se desgastan de manera irre- 
mediable (Ramírez, 2005). A esto se agrega que las prácticamente nulas barreras a la entrada y a la salida envían una señal de "invitación" a los entrantes potenciales, lo que reduce la demanda residual de cada una de las empresas establecidas y con ello sus ingresos. Lo anterior debilita a las empresas y deja su futuro a expensas de cualquier contingencia, como el fallo de algún implemento de la producción. No obstante, esta descapitalización muchas veces no es observada por el microempresario, más preocupado por obtener los ingresos que requiere para su subsistencia.

Por ello, estas empresas muchas veces prefieren no incurrir en innovaciones o mejoras al producto pues el mercado cautivo les asegura esos ingresos de subsistencia, por lo que no están dispuestas a comprometerse con un grado de diferenciación tal que les permita la venta de un extra en la producción ante la presencia de una demanda deprimida y un alto grado de rigidez en el flujo de clientes, los cuales ocupan el nivel más bajo en el escalafón de ingresos.

De acuerdo con los Censos Económicos de 2004 del Instituto Nacional de Estadística, Geografía e Informática (INEGI), las microempresas representan 95\% de la estructura industrial del país, y por falta de una política industrial que les dé certidumbre están siendo las perdedoras de los procesos de globalización y apertura que se han caracterizado por el trato igualitario a desiguales, lo cual ha agudizando la inequidad en el país (Mungaray y Ramírez, 2004), y especialmente en la zona fronteriza norte de México.

\section{Bibliografía}

Alcalá, C., V. De la O y E. Hernández, 2002, “Mercados y estrategias de comercialización en empresas pobres de Baja California", El Mercado de Valores, 52 (11): 12-19, Nacional Financiera, México, noviembre.

Barrón, K. y J. Aguilar, 2005, "Poder de mercado y marginación: el caso de Tijuana", en revista Encuentros, Universidad Autónoma de Nayarit, núm. 2, julio-diciembre, pp. 43-56. 
Cariola, C., M. Lacabana, L. Bethencourt, G. Darwich, G. Fernández y A. T. Gutiérrez, 1989, Crisis, sobrevivencia y sector informal, ILDISCENDES, Nueva Sociedad, Caracas, pp. 145.

Hualde, A., 2003, Aprendizaje e industria maquiladora. Análisis de las maquiladoras de la frontera del norte de México, Centro Interamericano de Investigación y Documentación sobre Formación, boletín 154, Formación en la empresa, en www.ilo.org/public/ spanish/region/ampro/cinterfor/publ/boletin/154/

Mungaray, A. y M. Ramírez, 2004, "Introducción a la asistencia universitaria para el desarrollo microempresarial", en Lecciones de microeconomía para microempresas, UABC, México, pp. 5-19.

Mungaray, A., N. Ramírez y M. Texis, 2005, “Estructura de mercado y maximización de beneficios en las microempresas", Comercio Exterior, 55 (4): 316-321, México.

Ocegueda, J., 2003, “Análisis kaldoriano del crecimiento económico de los estados de México, 1980-2000", Comercio Exterior, 53 (11), México.

Ochoa, H., 2005, “En Baja California hay confianza empresarial”, El Mexicano on line, en www.el-mexicano.com.mx/

Pickard, M., 2003, “Trans-Textil Internacional, S.A. de C.V., La Maquiladora de San Cristóbal de las Casas", en www.ecoportal.net/content/view/full/21338/.

Ramírez, N., 2005, Eficiencia y comportamiento de microempresas marginadas: una aplicación para los estados de Baja California y Nayarit, tesis doctoral, UABC, Facultad de Economía.

Ruiz, B. y P. Aceves, 2002, "Retos de frontera. Pobreza y desigualdad social en Tijuana", El Bordo, revista electrónica.

Segura, J., 1993, Teoría económica de la organización industrial, Civitas, Madrid, pp. 24-32.

Sierra, O. y S. Serrano, 2002, "Patrones y hábitos de consumo en Baja California", Comercio Exterior, 52 (8): 701-709, México.

Uriel, E., 1995, Análisis de datos. Series temporales y análisis multivariante, AC., Madrid, pp. 258-308. 


\begin{abstract}
ANEXO
a) El nivel de significancia es el estándar estadístico que se especifica para rechazar la hipótesis nula que indicaría igualdad en las medias de los grupos y, por lo tanto, que las variables independientes no ayudan a clasificar a los individuos en los distintos grupos de la variable dependiente. A lo largo del trabajo se especifica un nivel de significancia del $5 \%$, por lo que se rechaza la hipótesis nula solamente si se alcanza un nivel de confianza del 95\% para ello.

b) El grado de asociación entre las calificaciones discriminantes y los grupos está dado por la correlación canónica, la cual es una medida de la asociación entre la función discriminante (en caso de ser única, tal y como ocurre a lo largo de este documento) y el conjunto de variables simuladas que definen la membresía al grupo. Una correlación canónica alta indicaría que las variables discriminantes permiten diferenciar entre los grupos.

c) A través del estadístico lambda de Wilks se obtiene la relación entre las sumas de los cuadrados de las diferencias dentro de los grupos respecto a la suma total de cuadrados de las mismas. De esta forma, este estadístico expresa la proporción de variabilidad total no debida a las diferencias entre los grupos. Un valor cercano a 1 indica que las medias entre los grupos no parecen ser diferentes.

d) El porcentaje de aciertos indica la habilidad del modelo para agrupar correctamente los casos presentados con base en la muestra considerada.
\end{abstract}

